\title{
银杉花粉生命力及其变异
}

\section{王红卫 $^{1,2}$ 邓辉胜 ${ }^{1,3}$ 谭海明 ${ }^{4}$ 覃 琨 $^{4}$ 张定亨 5 葛 颂 $^{1 *}$ \\ (1 中国科学院植物研究所系统与进化植物学国家重点实验室 北京 100093) \\ （2 河南农业大学植物保护学院, 郑州 450002）（3 柳州师范高等专科学校, 广西柳州 545004) \\ （4 广西大瑶山国家级自然保护区管理局，广西金秀 545700）（5 花坪国家级银杉自然保护区，广西龙胜 541700)}

摘 要 中国特有植物银杉 (Cathay argyrophylla) 是最濒危的松杉类植物之一。研究表明, 受精前胚珠的高败育率 是银杉生殖效率低的重要原因之一, 但迄今对银杉花粉活力及其变异仍不得而知。由于花粉活力对授粉、受精、种 子产量和质量以及后代适合度都有直接影响，该研究采用 TTC 染色和体外萌发法测定了来自大瑶山 (Dayaoshan, DYS)和花坪 (Huaping, HP)两个地区 7 个种群 16 个个体 52 份银杉花粉样品的生活力。结果表明银杉花粉的活力水 平 $(93.3 \%$ ) 与其它裸子植物相当, 干燥低温条件下银杉的花粉活力比较稳定, 体外萌发的适宜蔗糖浓度在 $13 \%$ 左 右。ANOVA 分析揭示种群内个体间的花粉活力差异不大 $(p>0.05)$,而地区内的种群间及地区间的花粉活力有显 著差异 $(p<0.05)$ 。银杉花粉活力与其生殖成功没有相关性。在上述基础上，从控制授粉的角度讨论了银杉的保 护策略和复壮的可能性。

关键词 银杉 花粉活力 濒危 保护

\section{POLLEN VIABILITY AND VARIATION IN CATHAYA ARGYROPHYLLA}

WANG Hong-Wei ${ }^{1}{ }^{2}$, DENG Hui-Sheng ${ }^{13}$, TAN Hai-Ming ${ }^{4}$, QIN Kun $^{4}$, ZHANG Ding-Heng ${ }^{5}$, and GE Song $^{1 *}$

${ }^{1}$ State Key Laboratory of Systematic and Evolutionary Botany , Institute of Botany , Chinese Academy of Sciences , Beijing 100093 , China , ${ }^{2}$ College of Plant Protection, Henan Agricultural University , Zhengzhou 450002, China , ${ }^{3}$ Liuzhou Teachers College , Liuzhou , Guangxi 545004 , China , ${ }^{4}$ Dayaoshan Mountain National Nature Reserve , Jinxiu , Guangxi 545700 , China , and ${ }^{5}$ Huaping National Nature Reserve , Longsheng , Guangxi 541700 , China

\begin{abstract}
Aims Cathaya argyrophylla is one of the most endangered conifers endemic to China. Previous studies showed that this species was characterized by unusually low fertility and high embryonic mortality, which may be factors leading to its endangerment. Because knowledge of pollen viability is important for successful reproduction of plant species, we investigated pollen viability in natural populations of $C$. argyrophylla.

Methods We collected pollen samples from 16 individuals of seven populations in two locations, Dayaoshan (DYS) in Sichuan and Huaping (HP) in Guangxi. We evaluated pollen viability using the TTC method and analyzed its variation based on ANOVA.

Important findings Pollen viability of $C$. argyrophylla is comparable to other gymnosperms. Viability was stable in dry and low temperature conditions, and the optional sucrose concentration for pollen germination was about $13 \%$. We found low variation of pollen viability among individuals within populations $(p>0.05)$ but significant differentiation between populations within regions and among regions $(p<0.05)$. The pollen viability of $C$. argyrophylla is normal and thus not likely relevant to its endangerment. We proposed artificial crossings between genetically distinct populations as a potential conservation strategy for the population recovery of this endangered species.
\end{abstract}

Key words Cathaya argyrophylla , pollen viability , endangerment , conservation

\section{根据自然选择的基本原理，种群的进化速率与}

其遗传多样性成比例，也就是说 ，一个种群遗传多样

收稿日期 : 2006-07-21 接受日期 : 2007-01-20

基金项目 : 国家重点基础研究发展规划(973)项目 长江流域生物多样性变化、可持续性利用与区域生态安全” (G2000046805) 和中国科学 院知识创新工程重要方向项目 (KZCX2-YW-414)

感谢金秀大瑶山国家级银杉自然保护区的吴建生、兰光辉和唐广鹏及花坪国家级银杉自然保护区的刘宏等在花粉采集时的帮助

* 通讯作者 Author for correspondence E-mail : gesong@ibcas.ac.cn

E-mail of the first author : wghwcas@yahoo.com.cn 
性的下降意味着其适应环境变化的能力的下降 (Meffe \& Carroll , 1994)。而植物对环境的适应包括 植物发育各个阶段对环境的适应, 尤其是有性生殖 的各个阶段。花粉是雄配子体, 其活力和寿命对授 粉、受精、种子产量和质量以及后代适合度都有直接 影响 (Dafni \& Firmage, 2000 ; Ramsey \& Vaughton , 2000 ; Pflugshaupt et al., 2002 ; 李胜等, 2004)。例 如 张志勇和李德铢 (2003) 推测五针白皮松 (Pinus squamata) 极低的遗传多样性是结实率低、种子在野 外条件下难以萌发、进而导致种群面临衰竭处于濒 危境地的重要原因; 甘肃扁桃的研究表明低花粉活 力是其低坐果率的原因之一（李胜等，2004）; 通过 对德国特有植物 Cochlearia bavarica 控制授粉的研 究,Paschke 等(2002)发现花粉多态性越高繁殖成功 率也越高。另外, 许多研究也表明花粉活力和后代 适合度呈正相关 (Winsor et al. , 1987 ; Walsh \& Charlesworth , 1992 ; Mitchell , 1997)。

银杉 ( Cathaya argyrophylla) 是中国特有的松科 单型属植物。由于银杉起源古老, 分类地位独特, 在 松科系统发育重建和进化研究，以及在古生物、古气 候、古地质和古生态等方面的研究中都有重要科学 价值, 被誉为植物王国的 大熊猫”。据调查, 目前高 于 $1 \mathrm{~m}$ 的银杉个体数有 5000 余株 (谢宗强和李庆 梅, 2000) ,成熟个体的总数则不到 2000 株 (孙敏 等，1994）现存种群规模很小、孤立分布于相隔很 远的山地 (王伏雄，1990) ,成为最濒危的松杉类植 物之一(傅立国，1992 汪松和解炎，2004)。生殖生 物学研究表明, 银杉的结果率很低、球果的出种量也 很少, 空粒比例很高 (谢宗强等, 1998 ; 谢宗强和李 庆梅, 2000)。遗传多样性和谱系地理的研究也证 实了银杉的残遗特性，表现为低水平的遗传多样性 和强烈的种群间遗传分化 ${ }^{1)}$ (Ge et al. ,1998)。已有 的研究表明, 银杉受精前胚珠的高败育率可能是其 生殖效率很低的重要原因之一（谢宗强等，1998）。 然而, 迄今对银杉雄配子体的研究还很少, 银杉花粉 的活力是否正常, 是否影响到银杉的生殖成功, 以及 银杉花粉活力在个体间以及种群或地区之间是否有 差异等都不得而知。另一方面, 针对银杉种群遗传 多样性水平很低而种群间遗传分化明显, Ge 等 (1998)提出可以尝试采用传统的人工授粉方法, 在 不同种群的个体间、尤其是不同地区的个体间开展 杂交, 来恢复银杉的天然种群, 但这些工作需建立在
对银杉花粉生命力及其变异的了解的基础上。本研 究拟通过 TTC 染色法检测银杉花粉活力及其变异, 探讨花粉活力与银杉濒危的可能关系, 并为进一步 采取可能的保护和种群恢复措施提供技术基础。

\section{1 材料和方法}

\section{1 花粉取样}

2003 和 2004 年分别在广西金秀大瑶山 (Dayaoshan，DYS) 国家级自然保护区和龙胜花坪 (Huaping, HP) 国家自然保护区分别采集银杉花粉 样品。花粉样品以雄球花为单位, 将正在散粉的雄 球花轻轻取下, 放入纸袋内。在室内, 暴露空气中自 然干燥 $7 \mathrm{~d}$ 。除留存少量花粉进行连续活性检测外, 其余部分包于纸袋内, 硅胶干燥, 冰箱冷冻室保藏。 由于大瑶山的一些种群只有 $1 \sim 2$ 株成熟银杉个体, 雄球花极其稀少且不易采集,所以这些种群的个体 数和雄球花数都很少。最后的实验样品为来自大瑶 山(DYS)地区的 5 个种群和花坪 (HP) 地区的 2 个种 群, 每个种群取样个体为 $1 \sim 5$ 株不等, 每个个体的 取样雄球花为 $1 \sim 6$ 朵不等 (表 1 )。

用于检测花粉活力变化的 5 份样品分别来自大 瑶山 DYS1 (DYS1-1-1)、DYS3 ( DYS3-1-6 ) , DYS4 (DYS4-1-3) 以及 DYS5 (DYS5-1-1、DYS5-2-1) 种群。 而测试不同庶糖浓度下花粉萌发率的样品为大瑶山 的混合花粉。

\section{2 花粉活力检测}

花粉活力检测采用氯化三苯四氮唑 (TTC) 染色 法(胡适宜，1993)。氯化三苯四氮唑是一种氧化还 原色素, 水溶液无色, 遇到活细胞里的脱氢酶接受氢 离子, 还原后生成不溶于水的红色物质。花粉中呼 吸酶的活跃情况能正确地反映其生活力的强弱, 同 时氧化还原燃料能在呼吸酶的作用下着色, 因此可 根据着色情况判断花粉的生活力。该方法对许多植 物类群的花粉活力测定都很精确 (Lansac et al. , 1994)。干燥冷冻保藏的花粉检测活力前, 应作预处 理 :冷冻保存的花粉先在 $4{ }^{\circ} \mathrm{C}$ 冰箱内放置 $2 \sim 3 \mathrm{~h}$ 然 后在 $4{ }^{\circ} \mathrm{C}$ 冰箱里进行吸湿处理 $24 \mathrm{~h}$ 。制备染色装片 时 ,先在载玻片上加一滴 TTC 溶液 (配制方法见胡 适宜，1993）取少许花粉于其上，加盖玻片，然后置 恒温培养箱内, $37 \sim 38{ }^{\circ} \mathrm{C}$ 保温 (注意保持湿润)。 经连续检测发现, 保温 $1 \mathrm{~h}$ 左右染色花粉数稳定,且 比较容易区分出着色和非着色花粉粒 故以染色 $1 \mathrm{~h}$ 
为标准。 $16 \times 10$ 倍显微镜下检测 染成红色为有活 力花粉, 无色为无活力花粉 (花粉密集处染色很深, 散落周围的花粉染色很浅或不着色, 所以记数时, 选 取染色均匀, 花粉相对分散区域。随机选择 4 10 个视野, 计数花粉的总数和有生活力 (红色) 的花 粉数。每份样品计数花粉总数 $400 \sim 600$ 。按下式计 算有活力花粉的百分数：

有活力花粉百分数 $=($ 红色花粉数目 $/$ 花粉总 数) $\times 100 \%$

表 1 银杉花粉活力检测取样及检测结果

Table 1 Pollen samples of Cathaya argyrophylla collected in the study and their viability

\begin{tabular}{|c|c|c|c|}
\hline $\begin{array}{c}\text { 种群 } \\
\text { Population }\end{array}$ & $\begin{array}{c}\text { 个体 } \\
\text { Individual }\end{array}$ & $\begin{array}{l}\text { 雄球花数目 } \\
\text { No. of male } \\
\text { flowers }\end{array}$ & $\begin{array}{c}\text { 花粉活力范围 (平均值) } \\
\text { Pollen viability } \\
\text { ( average) }\end{array}$ \\
\hline 大瑶山 1(DYS1) & DYS1-1 & 4 & $84.4 \sim 92.8(87.9)$ \\
\hline 大瑶山 3(DYS3) & DYS3-1 & 3 & $89.3 \sim 92.7(91.5)$ \\
\hline \multirow[t]{2}{*}{ 大瑶山 4(DYS4) } & DYS4-1 & 5 & $86.7 \sim 93.0(90.1)$ \\
\hline & DYS4-2 & 1 & $\begin{array}{r}86.9 \\
88.5^{*}\end{array}$ \\
\hline \multirow[t]{5}{*}{ 大瑶山 5(DYS5) } & DYS5-1 & 3 & $85.4 \sim 86.9(87.7)$ \\
\hline & DYS5-2 & 3 & $90.1 \sim 95.2(92.4)$ \\
\hline & DYS5-5 & 6 & $85.9 \sim 93.4(90.9)$ \\
\hline & DYS5-6 & 5 & $89.1 \sim 92.9(91.2)$ \\
\hline & DYS5-8 & 3 & $\begin{array}{c}90.2 \sim 95.9(90.3) \\
90.5^{*}\end{array}$ \\
\hline 大瑶山 7(DYS7) & DYS7-1 & 1 & 98.1 \\
\hline $\begin{array}{l}\text { 大瑶山总数或平均 } \\
\text { Total or average in } \\
\text { DYS }\end{array}$ & 10 & 34 & 91.3 \\
\hline \multirow[t]{4}{*}{ 花坪 1(HP2) } & HP2-1 & 3 & $95 \sim 96(95.7)$ \\
\hline & HP2-2 & 3 & $92 \sim 96(94.0)$ \\
\hline & HP2-3 & 3 & $92 \sim 95(93.3)$ \\
\hline & & & $94.3^{*}$ \\
\hline \multirow[t]{4}{*}{ 花坪 2(HP3) } & HP3-1 & 3 & $96(96)$ \\
\hline & HP3-2 & 3 & $95 \sim 97$ (96) \\
\hline & HP3-3 & 3 & $95 \sim 97$ (96) \\
\hline & & & $96^{*}$ \\
\hline $\begin{array}{l}\text { 花坪总数或平均 } \\
\text { Total or average } \\
\text { in HP }\end{array}$ & 6 & 18 & 95.2 \\
\hline $\begin{array}{l}\text { 物种总数或平均 } \\
\text { Total or average } \\
\text { in species }\end{array}$ & 16 & 52 & 93.3 \\
\hline
\end{tabular}

* 种群平均花粉活力 Average value of pollen viability

离体条件下, 大多数植物的花粉在适宜的培养 基和温度条件下能够萌发。在 $20 \mathrm{ml}$ 雉形瓶内加入 $8 \mathrm{ml}$ 左右萌发花粉的溶液 (胡适宜，1993) ,取适量 花粉于瓶内。加塞防止水分蒸发。室温 (24 28 ${ }^{\circ} \mathrm{C}$ ) 条件下, 每天取样镜检, 有花粉管突起的花粉即 为萌发花粉。银杉花粉萌发缓慢, 大部分花粉管长 度超过花粉直径需 5 6 d 时间。由于花粉体外萌
发时霉菌污染严重，第四天时已不能制作装片，故以 第三天的萌发情况为最终结果。随机选取若干视 野, 计数花粉总数和萌发花粉数。每份样品计数至 少 300 粒花粉。按下式计算萌发率：

萌发率 $=$ (萌发花粉粒数 $/$ 花粉总数 $) \times 100 \%$

\section{3 数据分析}

应用 SPSS 10.0.1 软件分别从个体、种群和地区 水平上对银杉花粉活力进行统计分析。用于统计分 析的花粉活力以雄球花为单位, 包括所有采集到的 正常花粉样品。

\section{2 实验结果}

\section{1 银杉花粉寿命和萌发适宜蔗糖浓度}

对来自大瑶山 4 个种群 5 份花粉样品的活力检 测显示, 花粉采集后第七天, 有活性花粉比例平均降 低 5.2\%(图 1)。活力降低幅度最大的是 DYS1-1-1 $(8.3 \%)$, DYS5-1-1 降低幅度最小 $(2.3 \%)$ 。DYS4-13 和 DYS5-2-1 的活力下降幅度都未超过 5\% ,DYS31-6 样品的下降幅度为 $6.9 \%$ 。 $45 \mathrm{~d}$ 后, 花粉活力平 均下降 $6.5 \%$,DYS1-1-1 的下降幅度远高于其它样 品，达 12.4\%，DYS3-1-6、DYS4-1-3、DYS5-1-1 和 DYS5-2-1 分别下降 $6.6 \% 、 5.6 \% 、 2.5 \%$ 和 $4.9 \%$ 。 随后花粉活力没有明显的下降, 直至 $390 \mathrm{~d}$ 时仍维 持在较高的活力水平 ( $80 \%$ 以上)。这说明在干燥和 低温䛎藏的条件下, 银杉的花粉活力比较稳定。这 一结果为远距离的异地杂交和花粉的长期保存提供 了保障。
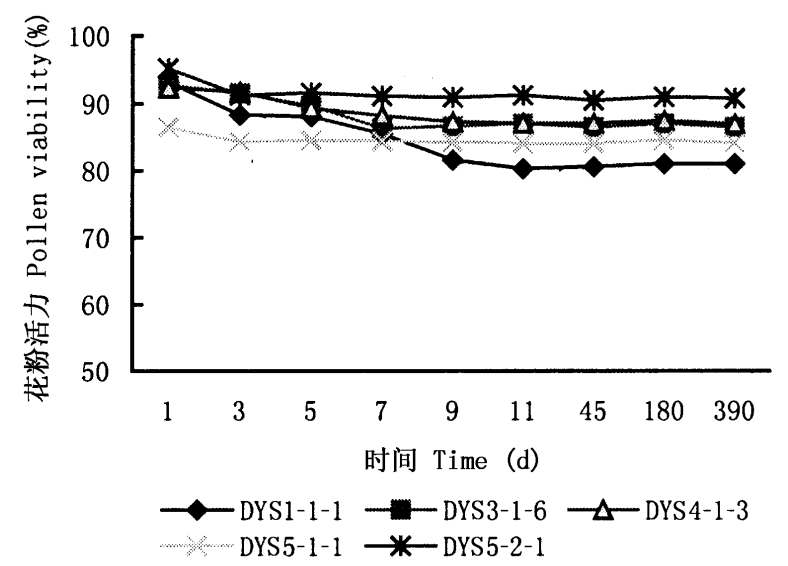

图 1 花粉活力随时间的变化

Fig. 1 The change of pollen viability with time

花粉样品来自大瑶山, 第一个数字代表种群, 第二个数字代表个 体, 第三个数字代表雄球花。例如 DYS5-2-1 是指大瑶山地区 5 号种 群 2 号样树的 1 号雄球花 Pollen samples come from Dayaoshan. The first number stands for population, the second for individual, the third for male flower. For example, DYS5-2-1 stands for male flower 1 of tree 2 in population 5 
离体萌发测定花粉生活力要得到正确的结果， 必须提供花粉萌发最适宜的条件。主要是营养培养 基和温度条件。一般来说, 各种植物在 $25{ }^{\circ} \mathrm{C}$ 左右都 是合适的, 但对培养基的要求不同物种有所不同, 特 别是起维持渗透压作用的蔗糖浓度 (胡适宜, 1993)。从多次萌发的结果来看, 银杉花粉萌发率较 低(未显示)。大瑶山混合花粉样品的检测表明, 银 杉花粉在 9\% 16\%蔗糖浓度范围内均有较高萌发 率, 低于 9\% 或高于 $16 \%$ 时萌发率大幅度下降 (图 2 )。蔗糖浓度为 $13 \%$ 时花粉萌发率最高, 因此银杉 花粉体外萌发的适宜蔗糖浓度为 $13 \%$ 左右。

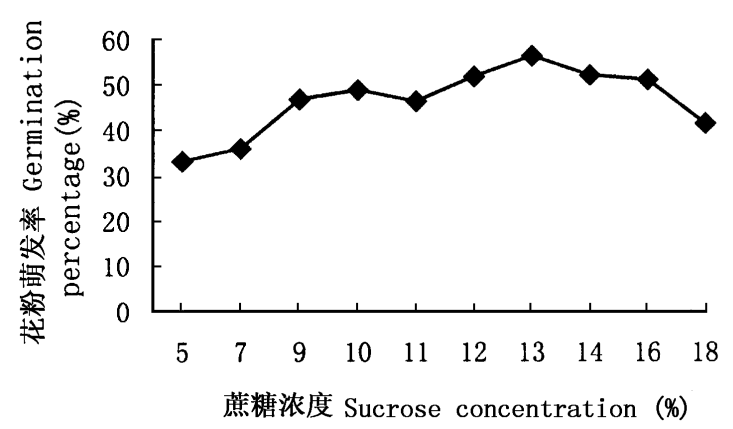

\footnotetext{
图 2 不同蔗糖浓度下银杉花粉萌发率 (大瑶山混合花粉)

Fig.2 Percentage of pollen germination at different sucrose concentrations (Mixed pollen grains from Dayaoshan)
}

\section{2 银杉花粉活力的个体变异和种群差异}

TTC 染色法鉴定的银杉花粉活力很高,有活力 的花粉数都在 $85 \%$ 以上 (表 1)。个体水平上,花粉 活力最小的是大瑶山的 DYS4-2, 为 $86.9 \%$, 最高者 是大瑶山的 DYS7, 为 $98.1 \%$,所有个体花粉活力的 平均值是 $93.3 \%$ 。物种范围内花粉活力个体变异 较大, 从 $86.9 \%$ 到 $98.1 \%$ 不等, 差异极显著 $(p=$ $0.000)$ 。两个地区内的个体变异有不同表现, 大瑶 山地区内的个体间有显著差异 $(p=0.042)$, 而花坪 地区内的个体差异不显著 $(p=0.059)$ 。同一种群 内的个体间差异较小, 都没有显著的个体差异 $(p>$ $0.05)$ 。

物种范围内 种群间花粉活力差异极显著 $(p<$ 0.001 ), 在 $87.9 \%$ 和 $98.1 \%$ 范围内变动 (表 1 )。大 瑶山地区, 各种群花粉活力变化很大 $(p<0.05)$, 最 高者为 $\operatorname{DYS7}(98.1 \%)$, 其次是 $\operatorname{DYS} 3(91.5 \%)$ 和 DYS5 $(90.5 \%)$, 最低的是 DYS1，只有 $87.9 \%$,最高 和最低的花粉活力相差 10 个百分点。花坪地区, 两 种群间的花粉活力也有较大差异 $(p<0.05), \mathrm{HP} 2$
为 $94.3 \%$, HP3 为 $96 \%$ 。平均水平上, 花坪的花粉活 力 $(95.2 \%)$ 高于大瑶山(91.3\%) (表 1) , 差异达显著 性水平。

\section{3 讨 论}

3.1 TTC 染色是一快速准确鉴定银杉花粉活力的 方法

TTC 染色是一快速 $(20 \sim 60 \mathrm{~min})$ 而准确的花粉 活力检测方法 (Lansac et al . 1994) ,已在许多植物 花粉活力研究中得到验证 (张子学和孙峰, 2002; 程 广有等, 1998 刘林德等,2001)。但该染色法的缺点 是颜色的界限不易判定，所以在应用时受到一定的 限制(Stone et al ., 1995)。本研究发现, 在用 TTC 对 银杉花粉染色时, 适当延长染色时间可获得清晰的 颜色分辨。此外, 染色后的银杉花粉颜色比较稳定, 可维持若干小时, 这与 Lansac 等(1994)的 $10 \mathrm{~min}$ 后 裉色结果截然不同。清楚的颜色判断、稳定的颜色 保持在高山松 ( Pinus densata) 花粉 TTC 染色中得到 佐证。因此,TTC 染色是快速、简便和有效的银杉雄 配子体活力检测方法。

\section{2 银杉具有同其它裸子植物类似的花粉活力}

通过 TTC 法测定的银杉有活力花粉比例很高, 个体花粉活力平均为 $93.3 \%$,与其它裸子植物花粉 活力相一致。采用同样的 TTC 染色法, 毛建丰等 (私人通讯) 检测出高山松的平均花粉活力也在 $90 \%$ 以上。梁建萍等 (2000) 应用活力估计偏高的次 甲基兰染色法研究了华北落叶松 (Larix principis-rupprechtii) 不同散粉期的花粉活力, 其最大值为 $93.5 \%$ 相当于银杉的平均水平。刚采集的银杏花 粉活力在 $92.9 \% \sim 98.2 \%$ 之间(王燕和张黎明, 2002）,与银杉的变化范围一致。然而, 与银杉的孤 立分布的濒危状况不同, 高山松在我国西南高山地 带呈广泛而连续的分布, 华北落叶松则是华北地区 中山以上的主要造林树种, 而银杏现已在我国广泛 栽培,成为许多城市的行道树。另外, 自然状态下, 银杉花粉活力在 $7 \mathrm{~d}$ 内没有明显的降低(图 1)。在 干燥低温条件下, 银杉花粉活力很稳定, 保藏一年后 仍有很高的活力指数。而同为裸子植物的东北红豆 杉( Taxus cuspidata) 花粉活力则有截然不同的表现 (程广有等, 1998)。与银杉花粉一样, 东北红豆杉 花粉先自然干燥, 然后在不同温度条件下保藏一段 时间后检测有生活力的花粉比例，检测方法同样采 用 TTC 染色。自然条件下, 东北红豆杉花粉咜藏 5 周时,有生活力的花粉仅占 $1.15 \%$,已基本丧失了 
生活力, 不能再用于杂交授粉。虽然低温塥藏可延 长花粉寿命, 但在 $-10{ }^{\circ} \mathrm{C}$ 咜藏 5 个月后, 有生活力 的东北红豆杉花粉也不足 5\%(程广有等，1998)。 这些结果表明, 与其它裸子植物相比, 银杉并未表现 出花粉活力的下降。与银杉雌配子体发育不正常相 比, 银杉雄配子发育并未出现异常, 不是导致其濒危 的主要原因。本研究结果也为远距离的异地杂交和 花粉的长期保存提供了科学依据。

\section{3 银杉花粉活力变异及其保护意义}

银杉的花粉活力表现出个体和种群间的变异, 尤其是在供试的两个地区间 (大瑶山和花坪)。银杉 花粉活力的差异可能是由于遗传因素的影响。等位 酶研究表明, 银杉种群间遗传分化很大 $\left(G_{\mathrm{st}}=\right.$ $0.441)$ 花坪的等位酶多态性 $(A=1.6 ; P=40.0$; $\left.H_{\mathrm{e}}=0.141\right)$ 高于金秀 $\left(A=1.3 ; P=28.0 ; H_{\mathrm{e}}=\right.$ 0.077)（Ge et al. , 1998)。这一遗传变异趋势在花 粉活力上得到了体现，花坪的花粉活力 (95.2\%) 显 著高于大瑶山 $(91.3 \%)(p<0.001)$ 。多基因序列数 据也表明银杉种群的遗传多态性变化很大, 种群间 存在显著性的遗传差异（Wang \& Ge，2006) , 但种群 的花粉活力和遗传多态性没有相关性。因此, 遗传 多样性水平和花粉活力之间的相关性还有待进一步 探讨。

Paschke 等 (2002) 对 Cochlearia bavarica 的研究 表明用多个外来种群的花粉进行授粉能提高结实率 和出种率。他们认为增加花粉源种群的数目就是提 高花粉多态性，这可使柱头受精时选择到最适宜的花 粉。从遗传角度看 花粉多态性是指花粉遗传多态性 的变异, 也既是种群间的遗传差异。Paschke 等 (2002) 的研究同时揭示小种群内授粉产生的后代较一般个 体要小, 并且很少能长成结果母树。他们推测这可能 是由于同一种群内的花粉多态性很低, 导致了后代适 合度的下降。Colling 等(2004)对菊科鸦憼属 Scorzonera humilis 的研究也得到同样的结果。由此看来, 在 进行银杉人工辅助授粉时, 除了考虑利用一定遗传差 异的种群花粉外，应尽可能选用多个外来种群的混合 花粉。然而, 需要注意的是开展遗传上有差异的种群 间杂交时可能会遇到异交衰退问题 (Waser \& Price , 1989 ; Fischer \& Matthies , 1997)。生态学研究表明，尽 管不同地点种群间的遗传差异很大, 但银杉生态需求 和生境特点却是类似的, 不存在明显的地方适应 (王 伏雄，1990; 谢宗强和陈伟烈，1999）。因此, 银杉种群 间的人工辅助授粉似乎切实可行, 但在具体实施前尚 需通过实验进行慎重评价。

\section{参 考 文 献}

Cheng GY (程广有), Tang XJ (唐晓杰), Yang ZG (杨振国), Sun HL (孙鹤林) (1998). Effect of different stocked temperature on pollen's life-span of Taxus. Journal of Jilin Forestry University (吉林林学院学报), 14, 196 - 198. (in Chinese with English abstract)

Colling G, Reckinger C, Matthies D (2004). Effects of pollen quantity and quality on reproduction and offspring vigor in the rare plant Scorzonera humilis (Asteraceae). American Journal of Botany, 91, $1774-1782$.

Dafni A, Firmage D (2000). Pollen viability and longevity: practical, ecological and evolutionary implications. Plant Systematics and Evolution, 222, $113-132$.

Fischer M, Matthies D (1997). Mating structure and inbreeding and out breeding depression in the rare plant Gentinanella germanica (Gentianaceae). American Journal of Botany, 84, $1685-1692$.

Fu LG (傅立国) (1992). China Plant Red Data Book: Rare and Endangered Plants Vol.1 (中国植物红皮书稀有濒危植物， 第一卷). Science Press, Beijing. (in Chinese)

Ge S, Hong DY, Wang HQ, Liu ZY, Zhang CM (1998). Population genetic structure and conservation of an endangered conifer, Cathaya argyrophylla (Pinaceae). International Journal of Plant Sciences, 159, $351-357$.

Hu SY (胡适宜) (1993). Experimental methods in plant embryology. I . Determination of pollen viability. Chinese Bulletin of Botany (植物学通报), 10,60-62. (in Chinese with English abstract)

Lansac AR, Sullivan CY, Johnson BE, Lee KW (1994). Viability and germination of the pollen of sorghum [Sorghum bicolor (L.) Moench]. Annals of Botany, 74, 27 - 33.

Li S (李胜), Li W (李唯), Yang DL (杨德龙), Wu JL (武季 玲), Cao ZY (曹孜义) (2004). Studies on examination of pollen vitality of almond and method for increasing fruit set rate. Journal of Fruit Science (果树学报), 21, 79-81. (in Chinese with English abstract)

Liang JP (梁建萍), Liang SF (梁胜发), Zhang FT (张风亭) (2000). A study on pollen of Larix principis - rupprechtii Mayr. Journal of Shanxi Agriculture University (山西农业大学学报), 20, 52-54. (in Chinese with English abstract)

Liu LD (刘林德), Zhang HJ (张洪军), Zhu N (祝宁), Shen JH (申家恒) (2001). Pollen viability and stigma receptivity of Eleutherococcus senticosus (Araliaceae). Bulletin of Botanical Research (植物研究), 21, 375-379. (in Chinese with English abstract)

Meffe GK, Carroll CR (1994). Principles of Conservation Biology. Sinauer Associates, Inc., Sunderland, Massachusetts.

Mitchell RJ (1997). Effects of pollen quantity on progeny vigor: evidence from the desert mustard Lesquerella fendleri. Evolution, 51, $1679-1684$.

Paschke M, Abs C, Schmid B (2002). Effects of population size 
and pollen diversity on reproductive success and offspring size in the narrow endemic Cochlearia bavarica (Brassicaceae). American Journal of Botany, 89, 1250 - 1259.

Pflugshaupt K, Kollmann J, Fischer M, Roy B (2002). Pollen quantity and quality affect fruit abortion in small populations of a rare fleshy-fruited shrub. Basic and Applied Ecology, 3, 319 327.

Ramsey M, Vaughton G (2000). Pollen quality limits seed set in Burchardia umbellata (Colchicaceae). American Journal of Botany, 87, $845-852$.

Stone JL, Thomson JD, Dent-Acosta SJ ( 1995 ) . Assessment of pollen viability in hand-pollination experiments: a review. American Journal of Botany, 82, 1186-1197.

Sun M(孙敏), Tang SH(汤绍虎), Chen AH(陈安和), Yang LY(杨兰英), Wang BN (王必浓) (1994). Studies on the growth of mature embryo of Cathaya argyrophylla in vitro and inducing of callus. Journal of Southwest Forestry College (西南林 学院学报), 14, 155 - 158. (in Chinese with English abstract)

Walsh NE, Charlesworth D (1992). Evolutionary interpretations of differences in pollen tube growth rates. The Quarterly Review of Biology, 67, $19-37$.

Wang FX (王伏雄) (1990). Biology of Cathaya argyrophylla (银 杉生物学). Science Press, Beijing. (in Chinese)

Wang S (汪松), Xie Y (解炎) (2004). China Species Red List Vol. 1 (中国物种红色名录, 第一卷). Higher Education Press, Beijing. (in Chinese)

Wang Y (王燕), Zhang LM (张黎明) (2002). Influences of storage temperatures on activated pollen ratio in Ginkgo biloba. Journal of Hubei Agricultural College (湖北农学院学报)，22，
213 - 214. (in Chinese with English abstract)

Waser NM, Price MV (1989). Optimal outcrossing in Ipomopsis aggregata : seed set and offspring fitness. Evolution, 43, 1097 1109.

Winsor JA, Davis LE, Stephenson AG (1987). The relationship between pollen load and fruit maturation and the effect of pollen load on offspring vigor in Cucurbita pepo. American Naturalist, $129,613-656$.

Xie ZQ(谢宗强), Chen WL(陈伟烈), Hu D(胡东), Zhu RG (朱日光) (1998). The fruiting characteristics of an endangered plant, Cathaya argyrophylla and the impact of animals on fruits. Acta Phytoecologica Sinica (植物生态学报), 22, 319-326. (in Chinese with English abstract)

Xie ZQ(谢宗强), Chen WL(陈伟烈) (1999). The endangering causes and preserving strategies for Cathaya argyrophylla, a plant endemic to China. Acta Phytoecologica Sinica (植物生态 学报), 23, 1-7. (in Chinese with English abstract)

Xie ZQ (谢宗强), Li QM (李庆梅) (2000). Seed characteristics of endangered plant Cathaya argyrophylla. Acta Phytoecologica Sinica (植物生态学报), 24, 82-86. (in Chinese with English abstract)

Zhang ZX (张子学), Sun F (孙峰) (2002). The selection of the best testing method on the pollen viability in hot pepper. Seed (种子) , 120, 32 - 33. (in Chinese with English abstract)

Zhang ZY(张志勇), Li DZ(李德铢) (2003). Conservation genetics of an extremely endangered pine, Pinus squamata. Acta Botanica Yunnanica (云南植物研究)，25，544-550。（in Chinese with English abstract)

责任编委 : 张大勇 责任编辑 : 李 敏 